\title{
Stimulation of murine P2Y11-like purinoreceptor protects against hypoxia/reoxygenation injury and decreases heart graft rejection lesions
}

\author{
Thierry Bourguignon, MD, ${ }^{\mathrm{a}, \mathrm{b}}$ Lauriane Benoist, $\mathrm{PhD},{ }^{\mathrm{a}}$ Stéphanie Chadet, $\mathrm{PhD},{ }^{\mathrm{a}}$ \\ Elodie Miquelestorena-Standley, $\mathrm{MD},{ }^{\mathrm{a}, \mathrm{c}}$ Gaëlle Fromont, $\mathrm{PhD},{ }^{\mathrm{c}}$ Fabrice Ivanes, $\mathrm{PhD},{ }^{\mathrm{a}, \mathrm{d}}$ and \\ Denis Angoulvant, $\mathrm{PhD}^{\mathrm{a}, \mathrm{d}}$
}

\begin{abstract}
Objective: Myocardial ischemia reperfusion is a major cause of cell injury during cardiac transplantation and is responsible for increased graft rejection. Several in vitro studies demonstrated the protective effect of P2Y11-like purinoreceptor stimulation in the context of myocardial ischemia/reperfusion. In this study, we hypothesized a possible cardioprotective role of P2Y11R stimulation against ischemia/reperfusion lesions and validated its clinical effect in vivo in a heart transplantation model.
\end{abstract}

Methods: We subjected $\mathrm{H} 9 \mathrm{c} 2$ rat cardiomyocyte-derived cell line to 5 hours of hypoxia and 1 hour of reoxygenation. P2Y11R selective agonist NF546 and antagonist NF340 were added at the onset of reoxygenation. Cell injuries were assessed by microculture tetrazolium reduction and intracellular adenosine triphosphate level. Clinical effect of P2Y11R stimulation was further investigated in vivo. Hearts from $\mathrm{BALB} / \mathrm{c}$ mice were transplanted intra-abdominally into allogenic C57BL/6 mice $(n=104)$. Recipient mice were injected with P2Y11R agonist. Mice in the sham group were injected with saline solution. In the control group, hearts from C57BL/6 were transplanted into syngeneic C57BL/6 mice. Rejection lesions were investigated using histology and immunohistochemistry at days 3, 5, and 7 after transplantation. We measured caspase activities to quantify apoptosis. Production of proinflammatory and anti-inflammatory cytokines was investigated.

Results: P2Y11R stimulation at the onset of reoxygenation significantly reduced in vitro hypoxia/reoxygenation injuries. This protection was suppressed with P2Y11R antagonist. In vivo, cardiac allograft survival was significantly prolonged after P2Y11R stimulation. Rejection lesions, classified according to the International Society of Heart Lung Transplantation guidelines and quantified using the mean number of inflammatory cells per field, were significantly reduced in the treated group. At day 5 after transplantation, P2Y11R agonist pretreated allografts also demonstrated less apoptotic lesions.

Conclusions: Our data suggest a novel cardioprotective role of P2Y11R at the onset of reoxygenation/reperfusion against reperfusion injuries. Pharmacologic conditioning using P2Y11 agonist may be beneficial after cardiac transplantation in improving myocardial ischemia/reperfusion outcomes and decreasing graft rejection lesions. (J Thorac Cardiovasc Surg 2019;158:780-90)

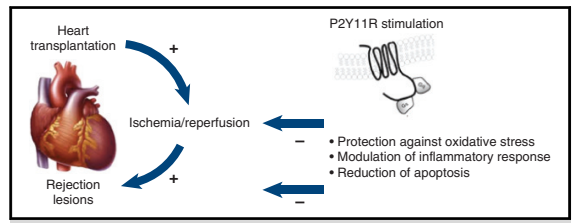

Therapeutic potential of P2Y11R in heart transplantation. During cardiac transplantation, myocardial ischemia reperfusion is a major cause of cell injury and is responsible for increased graft rejection. The P2Y11R is a G-protein coupled receptor of the P2Y receptor family. Our data suggest a beneficial role of P2Y11-like receptor stimulation in cardiac allograft conditioning through 3 main mechanisms: protection against oxidative stress, modulation of the inflammatory response, and reduction of apoptosis.

\section{Central Message}

P2Y11-like receptor stimulation by its specific agonist NF546 protects against H/R injuries, improves allograft survival, and decreases rejection lesions.

\section{Perspective}

Because current immunosuppressive regimens are still associated with multiple complications, including cancer, opportunistic infections, diabetes mellitus, kidney failure, and hypertension, short-term P2Y11-like receptor targeting at the time of transplantation may prove to be valuable in the clinical setting by promoting cardiac transplant tolerance and preventing rejection lesions.

See Commentary on page 791.

\footnotetext{
From the a EA4245 Transplantation, Immunologie et Inflammation, Loire Valley Cardiovascular Collaboration and FHU SUPORT, Tours University, Tours, France; ${ }^{\mathrm{b}}$ Department of Cardiac Surgery, Tours University Hospital, Tours, France; ${ }^{\mathrm{c}}$ Department of Pathophysiology, Tours University Hospital and Tours University, Tours, France; and ${ }^{\mathrm{d} D e p a r t m e n t ~ o f ~ C a r d i o l o g y, ~ T o u r s ~ U n i v e r s i t y ~ H o s p i t a l, ~ T o u r s, ~}$ France.

This work was supported by grants from the Société Française de Cardiologie, the Agence de la Biomédecine, and the Société Française de Chirurgie Cardio-Thoracique.
}

\footnotetext{
Part of this work was read as an abstract at the American Heart Association, November 12-16, 2016, New Orleans, Louisiana (Circulation. 2016;134:A15001). Received for publication July 10, 2018; revisions received Dec 4, 2018; accepted for publication Dec 4, 2018; available ahead of print Jan 30, 2019.

Address for reprints: Thierry Bourguignon, MD, Cardiac Surgery, Tours University Hospital, Centre, 37000 Tours, France (E-mail: thierry-bourguignon@hotmail.fr). $0022-5223 / \$ 36.00$

Copyright () 2018 by The American Association for Thoracic Surgery https://doi.org/10.1016/j.jtcvs.2018.12.014
} 

Abbreviations and Acronyms
ATP = adenosine triphosphate
DAMP $=$ damage-associated molecular pattern
DMEM = Dulbecco's modified Eagle's medium
DPBS = Dulbecco's phosphate-buffered saline
FBS = fetal bovine serum
$\mathrm{H} / \mathrm{R}=$ hypoxia/reoxygenation
HSC70 = heat shock cognate protein 70
IFN $=$ interferon
IL $=$ interleukin
$\mathrm{IQR}=$ interquartile range
$\mathrm{I} / \mathrm{R}=$ ischemia/reperfusion
ISHLT = International Society of Heart Lung Transplantation
MTT = methylthiazolyldiphenyl-tetrazolium
$\mathrm{P} 2 \mathrm{Y} 11 \mathrm{R}=\mathrm{P} 2 \mathrm{Y} 11$ receptor

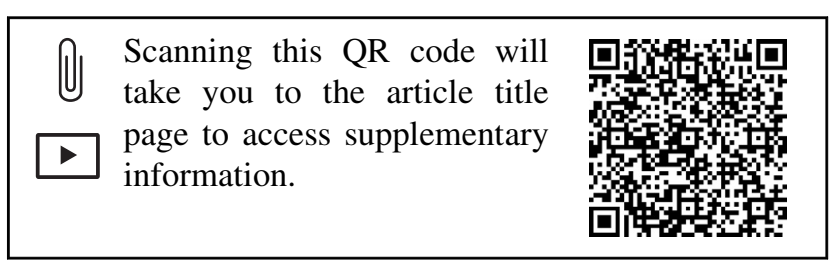

Heart transplantation is often the ultimate therapy for young patients with end-stage refractory heart failure. Its longterm outcomes, including chronic graft rejection, can be dramatically impaired by procedure-related myocardial ischemia/reperfusion (I/R) injuries. ${ }^{1} \quad$ Ischemia-related cellular stress is known to lead to irreversible tissue injuries. $^{2}$ Although shortening ischemia duration is mandatory to preserve organ function, ${ }^{3}$ sudden and massive reintroduction of oxygen and nutrients at the time of reperfusion further increases cell injuries and cardiomyocyte death through several mechanisms involving excessive oxidative stress. ${ }^{4}$ Cytoplasmic membrane rupture consecutive to $\mathrm{I} / \mathrm{R}$ injuries leads to release of damage-associated molecular patterns (DAMPs) within the extracellular space, which will activate immune cells and initiate an inflammatory response to I/R. Among DAMPs, extracellular purines (adenosine triphosphate [ATP], adenosine diphosphate, adenosine) and their purinergic receptors are important cardiovascular regulators. ${ }^{5}$ They may influence I/R injuries by several different mechanisms, including neutrophil migration, ${ }^{6}$ inflammasome activation, ${ }^{7,8}$ and dendritic cell maturation. ${ }^{9,10}$ So far, adenosine diphosphate receptor P2Y12 antagonists, which predominantly inhibit platelet aggregation, are the only used in clinic. In patients with acute coronary syndrome without ST-segment elevation, treatment with clopidogrel thus reduces the risk of myocardial infarction and recurrent ischemia. ${ }^{11}$ However, several other $\mathrm{P} 2$ receptors may be possible drug targets.

The P2Y11 receptor (P2Y11R) is a G-protein coupled receptor of the P2Y receptor family. Binding of its ligand (ATP) activates both adenylyl cyclase and phospholipase C via $\mathrm{Gs}$ and $\mathrm{Gq}$ coupling, a unique feature for a member of the P2Y receptor family. ${ }^{12,13} \mathrm{P} 2 \mathrm{Y} 11 \mathrm{R}$ is present in the myocardium and endothelium. ${ }^{14,15}$ Although several in vitro studies have suggested a potential cardioprotective effect of P2Y11R stimulation, in the context of myocardial infarction, ${ }^{16}$ little is known in the setting of heart transplantation.

In this study, we hypothesized that P2Y11R stimulation may decrease $\mathrm{I} / \mathrm{R}$ lesions associated with heart transplantation. We demonstrated in vitro its beneficial effect on cell death in the rat cardiomyoblast cell line H9c2 and further validated its clinical effect in vivo on rejection lesions using a murine heterotopic heart transplantation model.

\section{MATERIALS AND METHODS \\ Reagents}

ATP, $\mathrm{H}_{2} \mathrm{O}_{2}$, methylthiazolyldiphenyl-tetrazolium (MTT) bromide for microculture tetrazolium assay, and chemicals were purchased from Sigma-Aldrich (St Louis, Mo); P2Y11R agonist NF546 and antagonist NF340 were purchased from R\&D Systems (Minneapolis, Minn). Drugs were prepared in Gibco Dulbecco's phosphate-buffered saline (DPBS) solution with $\mathrm{CaCl}_{2}$ and $\mathrm{MgCl}_{2}$ from Fisher Scientific (Waltham, Mass).

\section{In Vitro Experiment}

Cell culture. H9c2 cells were obtained from American Type Culture Collection (LGC Standards) and cultured in Gibco Dulbecco's modified Eagle's medium (DMEM) supplemented with $10 \%$ fetal bovine serum (FBS) (HyClone; GE Healthcare Life Sciences, Marlborough, Mass) and penicillin-streptomycin $\left(100 \mathrm{U} / \mathrm{mL}\right.$, Gibco) at $37^{\circ} \mathrm{C}$ in a humidified incubator with $5 \% \mathrm{CO}_{2}$.

Hypoxia/reoxygenation simulation model. Hypoxia/reoxygenation $(\mathrm{H} / \mathrm{R})$ was simulated in vitro by oxygen and nutrient deprivation as previously described. ${ }^{10}$ After a 15-minute treatment with NF546 $10 \mu \mathrm{mol} / \mathrm{L}$ or NF340 $10 \mu \mathrm{mol} / \mathrm{L}$ in FBS-free DMEM, cells were placed for 5 hours in DPBS in a hypoxic chamber $\left(\mathrm{INVIVO}_{2} 200\right.$; Ruskinn Technology, Bridgend, UK) with $1 \% \mathrm{O}_{2}, 94 \% \mathrm{~N}_{2}$, and $5 \% \mathrm{CO}_{2}$, and then reoxygenated in a normoxic incubator $\left(5 \% \mathrm{CO}_{2}\right)$ for 1 hour in DMEM with vehicle or treatments. Control cells were left in a normoxic incubator in FBS-free DMEM for the same durations.

Assessment of cardiomyocyte viability and death. MTT reduction by mitochondria and CellTiter-Glo pLuminescent Assay (Promega, Madison, Wis) that measures intracellular ATP production were used as indicators of cell viability. After $\mathrm{H} / \mathrm{R}$ sequences, cells were placed in an MTT solution $(0.5 \mathrm{mg} / \mathrm{mL}$ in DMEM with $5 \%$ FBS $)$ for 1 hour at $37^{\circ} \mathrm{C}$ in a humidified incubator with $5 \% \mathrm{CO}_{2}$. The resulting formazan was solubilized in DMSO for 45 minutes. Absorbance was recorded at $550 \mathrm{~nm}$ using a microplate reader. CellTiter-Glo experiments were performed according to manufacturer's instructions using CellTiter-Glo diluted in DPBS (1/1) for 10 minutes at $37^{\circ} \mathrm{C}$. Resulting luminescence was measured with a GloMax-Multi Jr luminometer (Promega).

\section{In Vivo Experiment}

Animals. BALB/c and C57BL/6 mice ( $\mathrm{n}=208$; 6-week-old female) were purchased from Janvier LABS (Le Genest-Saint-Isle, France). All 
TABLE 1. List of antibodies used for Western blot

\begin{tabular}{|c|c|c|c|c|}
\hline Primary antibodies & Supplier & Species & Type & Reference \\
\hline P2Y11R & Alomone (Jerusalem, Israel) & Rabbit & Polyclonal & \#APR-015 \\
\hline Caspase-3 & $\begin{array}{l}\text { Cell Signaling Technology } \\
\text { (Danvers, Mass) }\end{array}$ & Rabbit & Polyclonal & 9508 \\
\hline Caspase- 9 & Cell Signaling Technology & Mouse & Monoclonal & 9662 \\
\hline Bax & Cell Signaling Technology & Rabbit & Polyclonal & 2772 \\
\hline Bcl-2 (50E3) & Cell Signaling Technology & Rabbit & Monoclonal & 2870 \\
\hline HSC70 & $\begin{array}{l}\text { Santa Cruz Biotechnology } \\
\text { (Dallas, Tex) }\end{array}$ & Mouse & Monoclonal & Sc-7298 \\
\hline \multicolumn{5}{|l|}{ Secondary antibodies } \\
\hline Rabbit-HRP conjugate & BioRad (Hercules, Calif) & Goat & Polyclonal & $\# 170-6515$ \\
\hline Mouse-HRP conjugate & BioRad & Goat & Polyclonal & \#170-6516 \\
\hline
\end{tabular}

HRP, Horseradish peroxidase; HSC70, heat shock cognate protein 70.

animal experiments were performed according to the 2010/63/UE directive on the protection of animals used for scientific purposes and accepted by the French Ministry of Agriculture. Sample size was calculated on the basis of power analysis, using an alpha risk of $5 \%$ and a power of $80 \%$.

Mouse heterotopic heart transplantation. Hearts from $\mathrm{BALB} / \mathrm{c}$ mice were transplanted intra-abdominally into allogenic C57BL/6 mice as previously described by Niimi (Video 1). ${ }^{17}$ Mice were anesthetized with a combination of ketamine $(100 \mathrm{mg} / \mathrm{kg})$ and xylazine $(10 \mathrm{mg} / \mathrm{kg})$ injected intraperitoneally. Briefly, donor ascending aorta and pulmonary trunk from the heart graft were anastomosed end-to-side to the recipient abdominal aorta and inferior vena cava, respectively, using 11-0 sutures. NF546 $10 \mu \mathrm{mol} / \mathrm{L}$ or vehicle (DPBS) was retro-orbitally injected in transplanted mice before starting the anastomoses. Graft survival, a surrogate marker of rejection, was evaluated by subcutaneous heartbeat palpation, and left ventricular contractility was assessed by echocardiography. Syngeneic transplantation (C57BL/6 mice) was used as control.

Cardiac histology and immunohistochemistry. Rejection lesions were investigated using histology and immunohistochemistry analysis (CD3, CD11c, CD45) in 36 allografts at days 3, 5, and 7 after transplantation.

Cardiac grafts were fixed in $10 \%$ paraformaldehyde, embedded in paraffin, cut into 5- $\mu \mathrm{m}$ sections, and stained with hematoxylin-eosin. For each slide, the mean number of inflammatory cells in 5 infiltrate foci (high-powered field) was measured using a $\times 40$ objective. Rejection lesions were also classified by 2 blinded experimented cardiac pathologists according to International Society of Heart Lung Transplantation (ISHLT) guidelines. ${ }^{18}$

For immunohistochemistry, harvested grafts were immediately frozen in optimal cutting temperature compound. Cryostat sections were fixed with acetone and stained with the following primary antibodies: antiCD3 (Abcam, Cambridge, UK), anti-CD11c (Anti-Mouse CD11c HL3 RUO; BD Biosciences, Franklin Lakes, NJ), and anti-CD45 (CD45 Antibody 30-F11; Biotechne, Minneapolis, Minn).

Western blot. H9c2 cells and cardiac grafts were lysed with RIPA lysis buffer containing $1 \%$ Triton X-100 and proteases inhibitors cocktail (Sigma-Aldrich) for 1 hour at $4^{\circ} \mathrm{C}$. Samples ( $20 \mu \mathrm{g}$ proteins) were loaded to Bolt 4-12\% Bis-Tris Plus gels (Invitrogen, Carlsbad, Calif) and transferred to Amersham Hybond PVDF membranes (GE Healthcare Life Sciences). The primary antibodies (Table 1) were incubated overnight at $4^{\circ} \mathrm{C}$. Membranes were further incubated for 1 hour at room temperature with appropriate secondary antibodies. Proteins were detected with Amersham ECL Prime reagent (GE Healthcare Life Sciences) using a PXi/PXi Touch gel imaging system and quantified by densitometric analysis using Fiji software (distributed by ImageJ). ${ }^{19}$

Serum levels of cytokines. At day 5 post-transplantation, serum levels $(\mathrm{pg} / \mathrm{mL}$ ) of interferon (IFN)- $\gamma$, interleukin (IL)-4, IL-5, IL-6, IL10, IL-12(p70), IL-13, IL-17A, IL-17F, IL-22, IL-33, and tumor necrosis factor- $\alpha$ were assessed using LEGENDplex Custom panel assay kit according to the instructions of the manufacturer (BioLegend, San Diego, Calif). Samples were acquired on a flow cytometer (FACSCanto, BD Biosciences) and analyzed using LEGENDplex Data Analysis Software provided by the manufacturer (BioLegend).

Primary and secondary end points. Primary outcome was graft survival (in days). Secondary end point was graft rejection lesions classified according to ISHLT guidelines and quantified using the mean number of inflammatory cells per field.

\section{Statistical Analyses}

Continuous data are presented as mean values \pm standard deviation or medians with interquartile range (IQR). Statistical analyses were performed using GraphPad Prism 5.0f (GraphPad Software, San Diego, Calif) for Mac OS X (Apple, Cupertino, Calif). The Shapiro-Wilk test was used to determine whether a continuous outcome was well modeled by a normal distribution. The $t$ test was used for analyzing normally distributed continuous variables, and the Mann-Whitney $U$ test was used for non-normally distributed variables. Survival curves were compared using the log-rank test.

\section{RESULTS}

\section{Specific Stimulation of P2Y11-Like Receptor Decreases Hypoxia/Reoxygenation Injuries in Rat Cardiomyocytes}

$\mathrm{H} / \mathrm{R}$ significantly decreased $\mathrm{H} 9 \mathrm{c} 2$ cells viability compared with normoxic control $(-34.7 \%$; IQR, 25.143.2; $P<.001 ; \mathrm{n}=7$ ) (Figure $1, A$ ). Despite the lack of rodent P2Y11R gene orthologous to human, many reports in the literature support the existence of a P2Y11-like receptor in these species. ${ }^{16,20,21} \mathrm{We}$ immunodetected a slightly less than $30 \mathrm{kDa}$ band in $\mathrm{H} 9 \mathrm{c} 2$ protein lysates using antiP2Y11 antibody, further supporting the existence of a P2Y11R-like protein in this rodent-derived cell line 


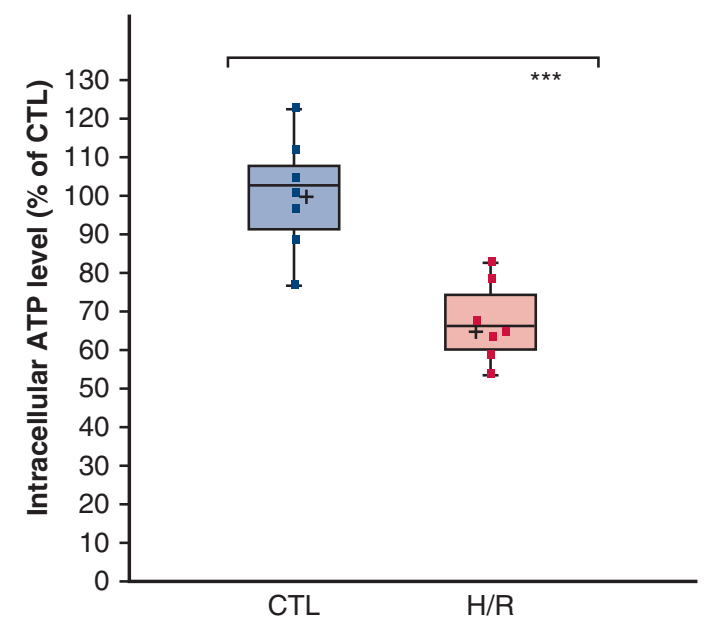

A

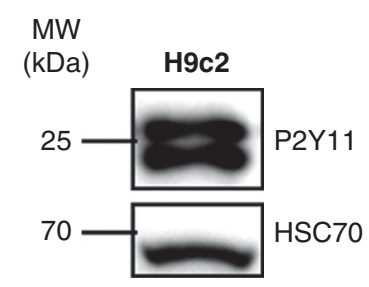

B

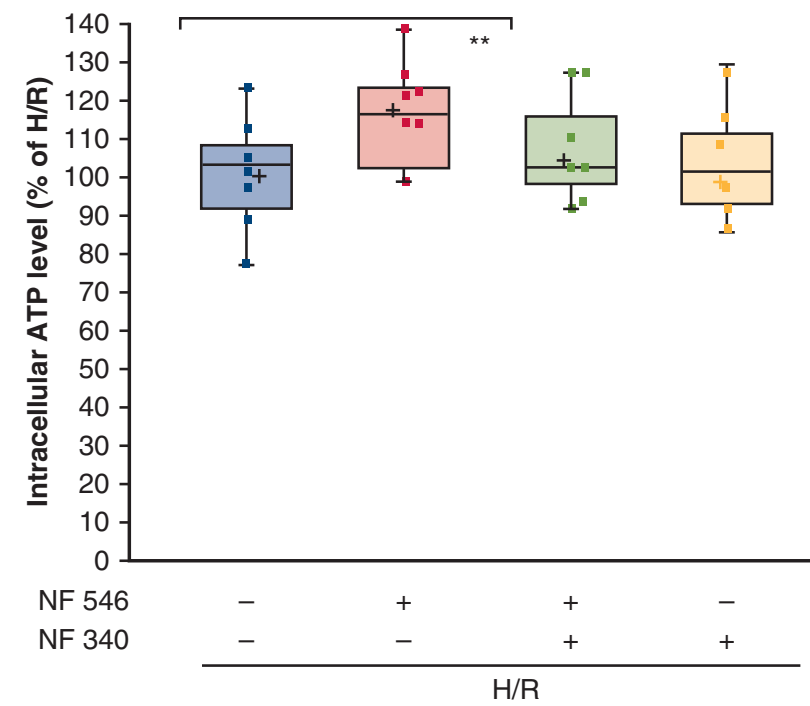

C

FIGURE 1. Cardioprotective effect of P2Y11-like receptor stimulation against $\mathrm{H} / \mathrm{R}$ lesions in $\mathrm{H} 9 \mathrm{c} 2$ cells. $\mathrm{H} 9 \mathrm{c} 2$ cells were subjected to 5 hours of hypoxia $\left(1 \% \mathrm{O}_{2}\right.$, DPBS solution) followed by 1 hour of reoxygenation $\left(21 \% 0_{2}, \mathrm{DMEM}\right)(\mathrm{H} / \mathrm{R})$. MTT reduction by mitochondria and intracellular ATP production were used as indicators of cell viability. A, H9c2 cells viability decreased after $\mathrm{H} / \mathrm{R}$ (red dots; $\mathrm{n}=7$ ) compared with control (blue dots; $\mathrm{n}=7$ ). B, P2Y11-like receptor expression analyzed by Western blot from total proteins of $\mathrm{H} 9 \mathrm{c} 2$ cells cultured in normoxia $(\mathrm{n}=3)$. The immunodetection of a slightly below $30 \mathrm{kDa}$ band in $\mathrm{H} 9 \mathrm{c} 2$ protein lysates confirms the existence of a P2Y11R-like protein in this rodent-derived $(\mathrm{n}=3)$ (Figure $1, B)$. Treatment of H9c2 cells with P2Y11 selective agonist, NF546 $(10 \mu \mathrm{mol} / \mathrm{L})$, at the time of reoxygenation significantly increased cell viability $(+17.3 \%$; IQR 12.1-23.2; $P<.01 ; \mathrm{n}=7)$, whereas co-treatment with P2Y11 antagonist NF340 $(10 \mu \mathrm{mol} / \mathrm{L})$ abolished this effect (Figure 1,C).

\section{P2Y11-Like Receptor Stimulation Increases Allograft Survival Time in a Murine Model of Heterotopic Heart Transplantation}

A total of 208 mice were used in the present study, allowing 104 transplantations. Hearts from $32 \mathrm{BALB} / \mathrm{c}$ mice were transplanted intra-abdominally into C57BL/6 recipients. Survival of allograft in P2Y11R agonist-treated recipients (treated group: $9.6 \pm 1.9$ days, $\mathrm{n}=16$ ) was significantly prolonged compared with that of vehicle-treated recipients (sham group: $8.2 \pm 1.4$ days, $\mathrm{n}=16 ; P=.04$ ). This represents a $17 \%$ increase in survival time. Syngeneic grafts (control group) from C57BL/6 mice $(n=8)$ showed no sign of rejection when recipients were killed at 6 weeks after transplantation (Figure 2).

\section{P2Y11-Like Receptor Stimulation Decreases Rejection Lesions}

Conventional histology allowed identification of acute graft rejection in 36 allografts (Figure 3). As depicted in Figure $3, A$, at postoperative day 3 , only discrete cardiac allograft infiltration was observed in both P2Y11R agonist-treated and vehicle-treated recipients, with a number of inflammatory cells count that averaged 76 (IQR, 67$89)$ and 81 (IQR, 76-94) cells, respectively $(P=.21)$. In contrast, at postoperative day 5 , the number of inflammatory cells was significantly lower in the treated group (122 [115-134] vs 196 [181-215] in the vehicle-treated group; $P<.05)$. All allografts in untreated recipients $(n=15)$ exhibited histologic evidence of grade $3 R$ acute rejection (ISHLT classification), whereas allografts in P2Y11R-treated recipients $(\mathrm{n}=15)$ were classified $1 \mathrm{R}$ or 2R (Figures 3, B, and 4). As expected, syngeneic grafts $(\mathrm{n}=3)$ showed no sign of rejection. Likewise, isolated

cell line $(\mathrm{n}=3)$. C, Treatment of H9c2 cells with P2Y11 selective agonist, NF546 $(10 \mu \mathrm{mol} / \mathrm{L})$, at the time of reoxygenation significantly increased cell viability $(+17.3 \%$; IQR, 12.1-23.2; $P<.01 ;$ red dots $; \mathrm{n}=7)$, whereas co-treatment with P2Y11 antagonist, NF340 (10 $\mu \mathrm{mol} / \mathrm{L})$, abolished this effect (green dots; $\mathrm{n}=7$ ). There was no significant difference using NF340 alone (yellow dots; $\mathrm{n}=7$ ). Data in A and C are presented as boxand-whiskers plots where the upper and lower borders of the box represent the upper and lower quartiles, the horizontal line inside the box represents the median, the upper and lower whiskers represent the maximum and minimum values of nonoutliers, and the + sign represents the mean. $* * P<.01$, $* * * P<.001$ compared with control condition (CTL/-). ATP, Adenosine triphosphate; $C T L$, control; $H / R$, hypoxia/reoxygenation. 


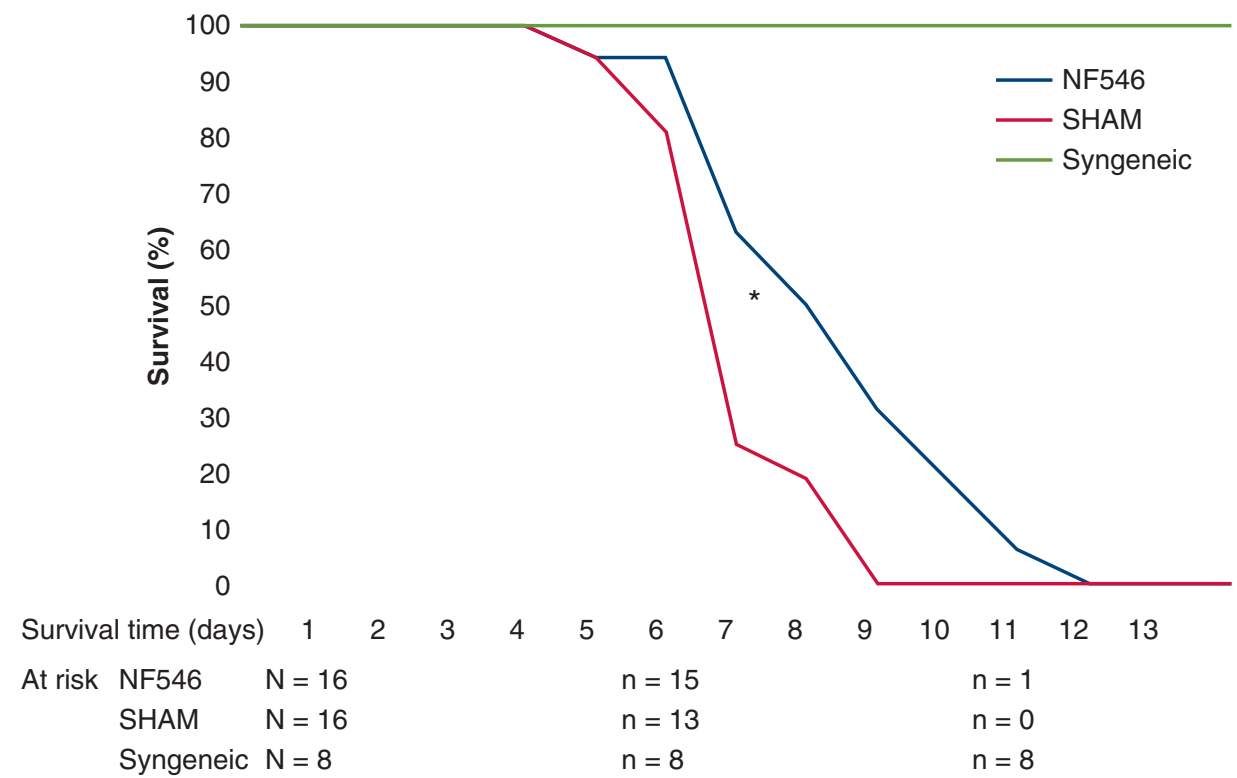

FIGURE 2. Graft survival in days after heart transplantation. Graft rejection was not observed in syngeneic grafts (green curve). Survival of P2Y11R agonist-treated allografts $(9.6 \pm 1.9$ days, $\mathrm{n}=16$; blue curve $)$ were significantly prolonged compared with that of untreated hearts $(8.2 \pm 1.4$ days, $\mathrm{n}=16 ; P=.04 ;$ red curve $) . * P<.05$.

retro-orbital injection of vehicle $(\mathrm{n}=3)$ did not result in significant inflammatory cells infiltration.

\section{P2Y11-Like Receptor Stimulation Decreases Cardiac Apoptosis}

At day 5, caspase- 3 activity was significantly increased in allogeneic grafts, compared with syngeneic grafts and isolated retro-orbital injection of vehicle, cleaved caspase-3/ heat shock cognate protein 70 (HSC70) ratio averaging 0.3 (IQR, $0.2-0.5)$ versus 0 and 0 , respectively $(P<.05$ vs syngeneic grafting; $P<.05$ vs isolated retro-orbital injection of vehicle). In allografts of P2Y11R agonist-treated recipients, this increased activity was significantly attenuated $(P<.05$ vs untreated recipient allografts) (Figure 5, $A)$.

Likewise, caspase- 9 activity was increased at day 5 in allogeneic grafts, compared with syngeneic grafts and isolated retro-orbital injection of vehicle, cleaved caspase-9/ HSC70 ratio averaging 0.4 (IQR, 0.3-0.5) versus 0.3 (IQR, 0.2-0.4) and 0.3 (IQR, 0.2-0.4), respectively $(P<.05$ vs syngeneic grafting; $P<.05$ vs isolated retroorbital injection of vehicle). However, this increased activity was not significantly attenuated by P2Y11R agonist treatment (Figure 5, B).

Expression level of Bax was not significantly increased in allogeneic grafts compared with syngeneic grafts and isolated retro-orbital injection of vehicle (Figure 6, A), whereas Bcl-2 expression was significantly decreased in allogenic grafts compared with syngeneic grafts and isolated retro-orbital injection of vehicle, relative $\mathrm{Bcl}-2 /$ HSC70 ratio averaging 0.2 (IQR, 0.1-0.2) versus
1.0 (IQR, 0.8-1.3) and $1.0(\mathrm{IQR}, 0.8-1.2)$, respectively $(P<.05$ vs syngeneic grafting; $P<.05$ vs isolated retroorbital injection of vehicle). Bax expression was not significantly modified by P2Y11R agonist treatment (Figure 6, $B$ ). $\mathrm{Bax} / \mathrm{Bcl}-2$ ratio was increased at day 5 in allogeneic grafts, compared with syngeneic grafts and isolated retro-orbital injection of vehicle, averaging 10.5 (IQR, 7.3-13.5) versus 1.1 (IQR, 0.3-1.5) and 1.0 (IQR, 0.3-1.5), respectively $(P<.05$ vs syngeneic grafting; $P<.05$ vs isolated retroorbital injection of vehicle). In allografts of P2Y11R agonist-treated recipients, this increased ratio was significantly attenuated $(P<.05$ vs vehicle-treated allografts) (Figure 6, C).

\section{P2Y11-Like Receptor Stimulation Decreases Inflammation After Heart Transplantation}

At day 5, the group treated with P2Y11R agonist $(n=6)$ demonstrated significantly lower proinflammatory cytokines IFN $\gamma$ and IL-12 serum levels, and significantly higher anti-inflammatory cytokine IL-10 levels compared with the vehicle-treated group $(\mathrm{n}=6)(P<.05)$. IL-4, IL-5, IL-6, IL-13, IL-17A, IL-17F, IL-22, IL-33, and tumor necrosis factor- $\alpha$ protein levels were not different among the experimental groups (Table E1).

\section{DISCUSSION}

In the present study, we demonstrated that P2Y11-like receptor stimulation decreased I/R lesions associated with heart transplantation. We investigated in vitro its beneficial effect on cell survival in the rat cardiomyoblast cell line 


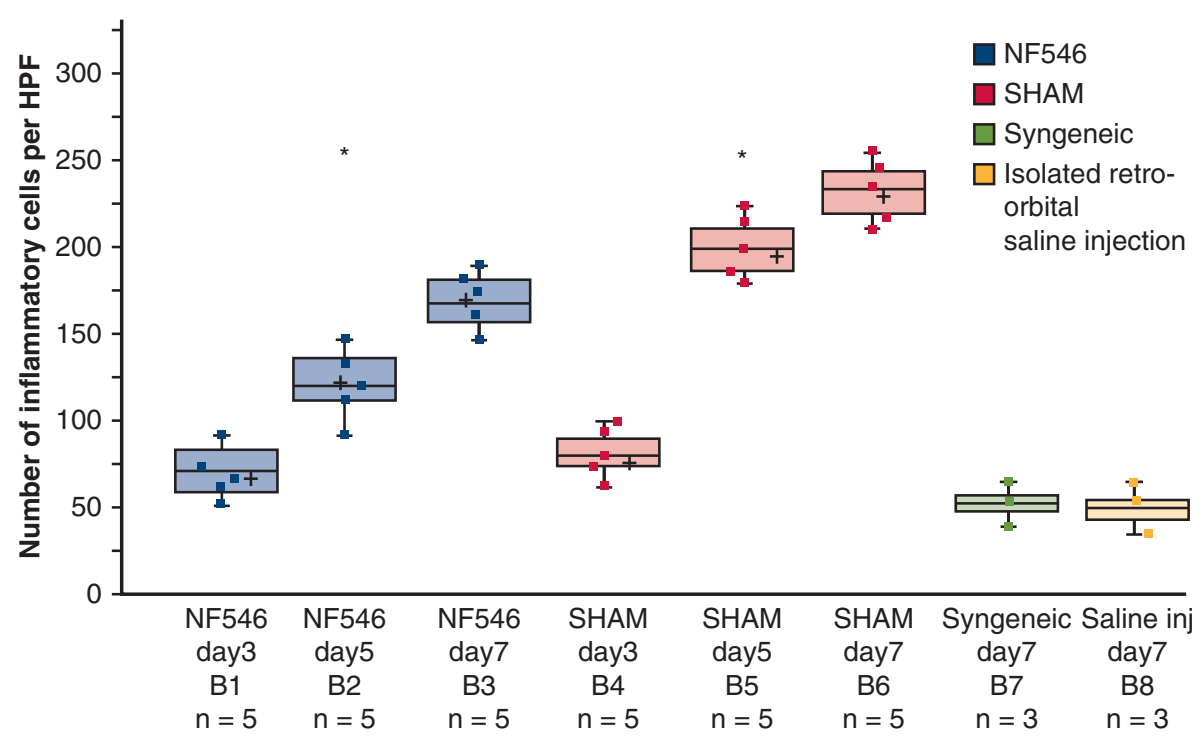

A
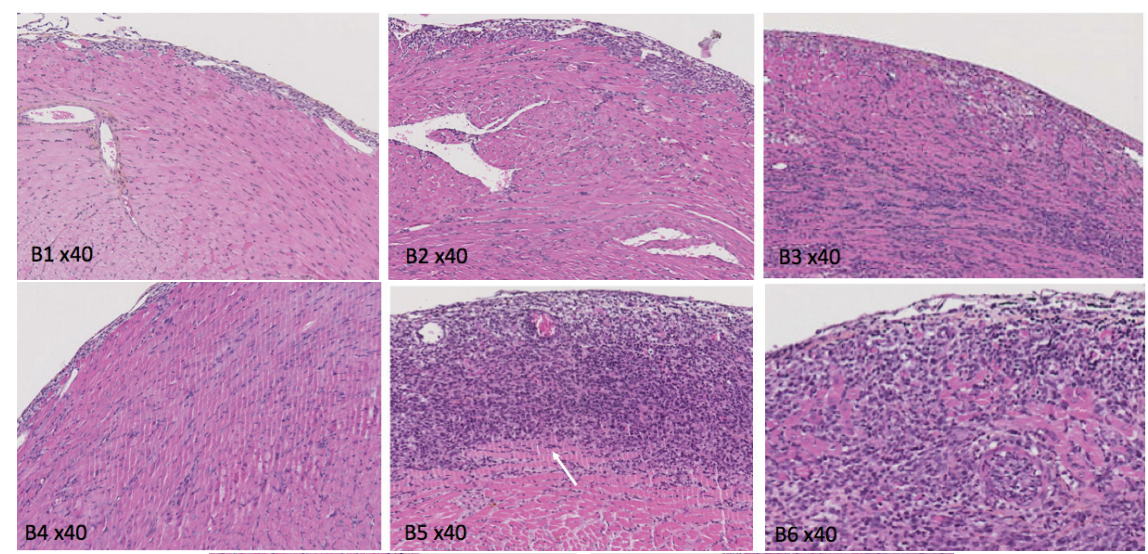

B
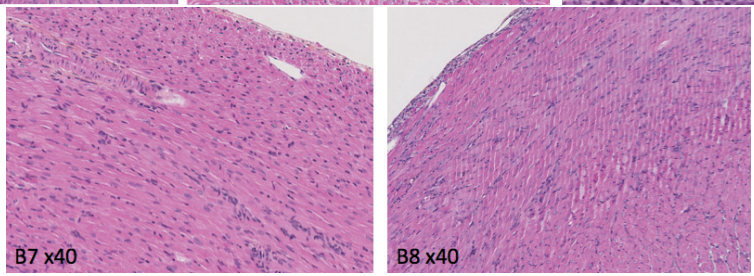

FIGURE 3. Acute graft rejection. A, Mean number of inflammatory cells per high power field $(\times 40)$ in grafted hearts at postoperative day 3,5 , or 7 . At postoperative day 5, number of inflammatory cells count was significantly lower in the treated group (122 [115-134]; blue plots vs 196 [181-215] in the vehicle-treated group; red plots; $P<.05)$. As expected, syngeneic grafts (green plots) and isolated retro-orbital injection of vehicle (yellow plots) did not result in significant inflammatory cells infiltration. Data in A are presented as box-and-whiskers plots where the upper and lower borders of the box represent the upper and lower quartiles, the horizontal line inside the box represents the median, the upper and lower whiskers represent the maximum and minimum values of nonoutliers, and the + sign represents the mean. ${ }^{*} P<.05$. B, Conventional histology in P2Y11R agonist-treated allografts (NF546) (B1-B3) and vehicle-treated allografts (SHAM) (B4-B6) at postoperative day 3, 5, and 7, respectively. None of the syngeneic grafts (B7) or native hearts with isolated retro-orbital injection of vehicle (B8) exhibited signs of rejection at day 7. At day 5, untreated allografts exhibited an increase of inflammatory cells with obvious myocyte damage and destruction of cardiac muscle fibers (arrow, B5) which were attenuated under P2Y11R agonist conditioning (B2). HPF, High-powered field. 


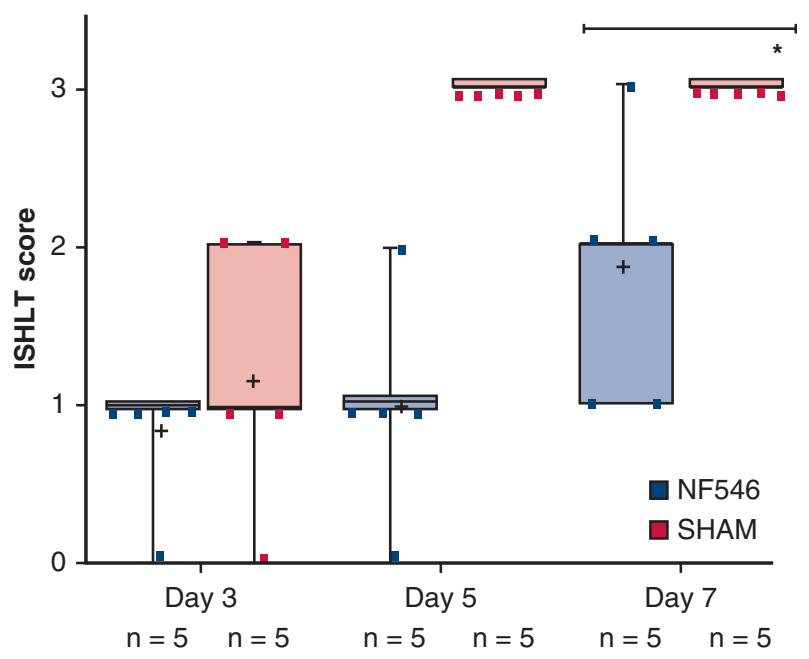

FIGURE 4. Acute rejection grading using ISHLT classification. Five days after transplantation, compared with vehicle-treated allografts (SHAM; red dots), P2Y11R agonist-treated mice (NF546; blue dots) demonstrated less foci of mononuclear cells (lymphocytes/macrophages) and less myocytes damages, resulting in lower ISHLT standardized cardiac biopsy grade. Data are presented as box-and-whiskers plots where the upper and lower borders of the box represent the upper and lower quartiles, the horizontal line inside the box represents the median, the upper and lower whiskers represent the maximum and minimum values of nonoutliers, and the + sign represents the mean. $* P<.05$. ISHLT, International Society of Heart Lung Transplantation.

$\mathrm{H} 9 \mathrm{c} 2$ and further validated its clinical effect in vivo on rejection lesions using a murine heterotopic heart transplantation model.

\section{P2Y11-Like Receptor Stimulation Protects Against Oxidative Stress}

The cardioprotective role of P2Y11R against myocardial $\mathrm{I} / \mathrm{R}$ injuries was previously suggested in several in vitro studies. Balogh and colleagues ${ }^{21}$ reported a positive inotropic effect in murine cardiomyocytes, whereas Amisten and colleagues ${ }^{22}$ demonstrated that a P2Y11R polymorphism (Ala-87-Thr) was associated with an increase in both serum inflammation and risk of myocardial infarction. More recently, Djerada and Millart ${ }^{16}$ also reported effective cardioprotection with P2Y11R pharmacologic preconditioning in rodent Langendorff hearts. We first confirmed these ex vivo data by studying $\mathrm{H} / \mathrm{R}$ injuries in $\mathrm{H} 9 \mathrm{c} 2$ cardiomyocytes and showed that $\mathrm{H} / \mathrm{R}$ defined as oxygen nutrients deprivation/resupply decreased $\mathrm{H} 9 \mathrm{c} 2$ viability compared with control cells. Activation of cell death programs induces the release of DAMPs, among which extracellular ATP (released during $\mathrm{I} / \mathrm{R}$ by suffering cardiomyocytes ${ }^{14,15}$ and endothelial cells ${ }^{23}$ ) is an important regulator of immune system activation through purinergic receptors (P2R) stimulation. ${ }^{3,24,25}$ By using NF546, a specific non-nucleotide agonist of $\mathrm{P} 2 \mathrm{Y} 11,{ }^{26}$ we showed for the first time that stimulation of P2Y11-like receptor at the onset of reoxygenation protected rat cardiomyoblasts $H 9 c 2$ against $H / R$ injuries. Our data are in keeping with those in a rat Langendorff model showing that nicotinic acid adenine dinucleotide phosphate pharmacologic preconditioning was cardioprotective through P2Y11R activation. ${ }^{16}$ In addition, an increased risk of myocardial infarction together with increased levels of inflammatory markers was observed in patients carrying P2Y11R polymorphism Ala-87-Thr. The proposed mechanism was a change in the 3-dimensional structure of the receptor interfering with ligand binding and signaling. ${ }^{22}$

As for the mechanisms involved in the cardioprotection mediated by P2Y11R against oxidative stress, preliminary data from our group suggested that its stimulation has a direct effect on reactive oxygen species production and cell resistance to inflammation and apoptosis. ${ }^{27}$ For example, protein kinase $\mathrm{C}$ epsilon is known to be activated by the phospholipase $\mathrm{C} /$ diacyl glycerol pathway subsequent to Gq protein-coupled receptor stimulation (eg, P2Y11R), leading to its translocation to mitochondria. ${ }^{21}$ This may be considered as a valuable target in cardioprotection because of its ability to interact with mitochondrial ATPsensitive $\mathrm{K}^{+}$channels subsequently modifying mitochondrial permeability transition. ${ }^{28}$

\section{P2Y11-Like Receptor Stimulation Increases Allograft Survival and Decreases Rejection Lesions After Heart Transplantation}

Late graft failure is the leading cause of late morbidity and mortality in heart transplant recipients. The International Society of Heart and Lung Transplantation registry in 2017 stated that 5 years after cardiac transplantation, late graft failure accounted for $24 \%$ of deaths. ${ }^{29}$ To our knowledge, the present study is the first to describe a cardioprotective effect of P2Y11R stimulation in vivo in a model of heart transplantation. Among animal transplantation models used to study $I / R$ injuries, the mouse is preferred because of the high degree of similarity between the mouse $\mathrm{H}-2$ major histocompatibility complex and the human leukocyte antigen system, which provides clinically relevant mechanistic insights. ${ }^{30}$ By using the specific nonnucleotide agonist NF546, we showed for the first time that conditioning with P2Y11R stimulation at the time of heart transplantation significantly increased allograft survival. Our histologic studies revealed that 5 days after transplantation, P2Y11R agonist-treated mice demonstrated fewer rejection lesions, for example, less foci of mononuclear cells (lymphocytes/macrophages) and myocyte damage, resulting in lower ISHLT standardized cardiac biopsy grade.

In regard to the mechanisms involved in P2Y11Rmediated cardioprotection against rejection lesions, several reports demonstrated the role of extracellular ATP as an 

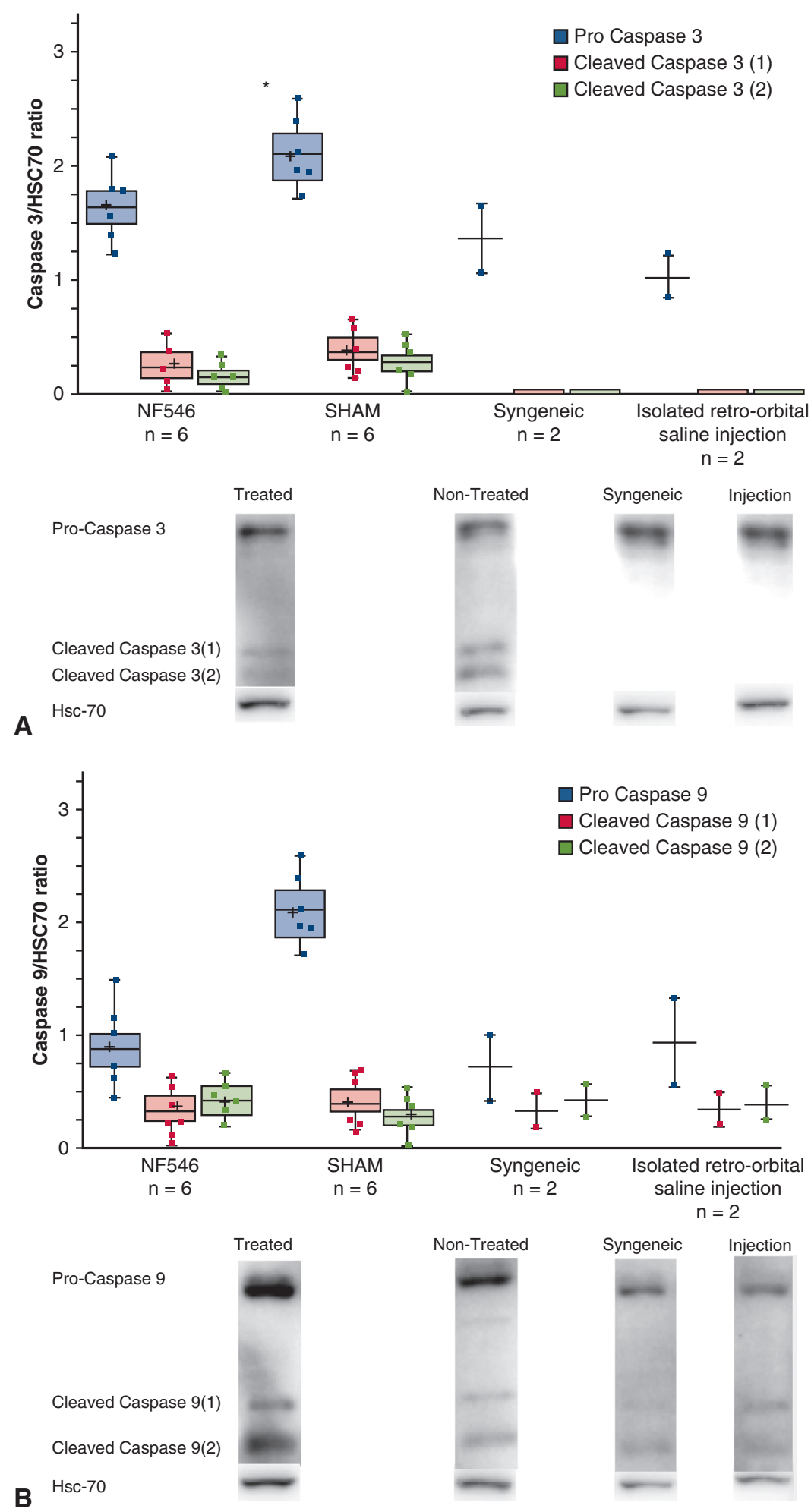

FIGURE 5. Quantification of caspase activity. Comparison of cleaved caspase-3/HSC70 (A), cleaved caspase- $9 /$ HSC70 (B), at day 5 in treated allografts (NF546; $n=6$ ), untreated allografts (SHAM; $n=6$ ), syngeneic grafts $(n=2)$, and mice undergoing isolated retro-orbital injection of vehicle $(n=2)$. Data are presented as box-and-whiskers plots where the upper and lower borders of the box represent the upper and lower quartiles, the horizontal line inside the box represents the median, the upper and lower whiskers represent the maximum and minimum values of nonoutliers, and the + sign represents the mean. P2Y11R agonist-treated allografts demonstrated a significant decrease in caspase- 3 activity compared with untreated allografts, whereas caspase- 9 activity was not significantly attenuated by NF546 treatment. $* P<.05$. 

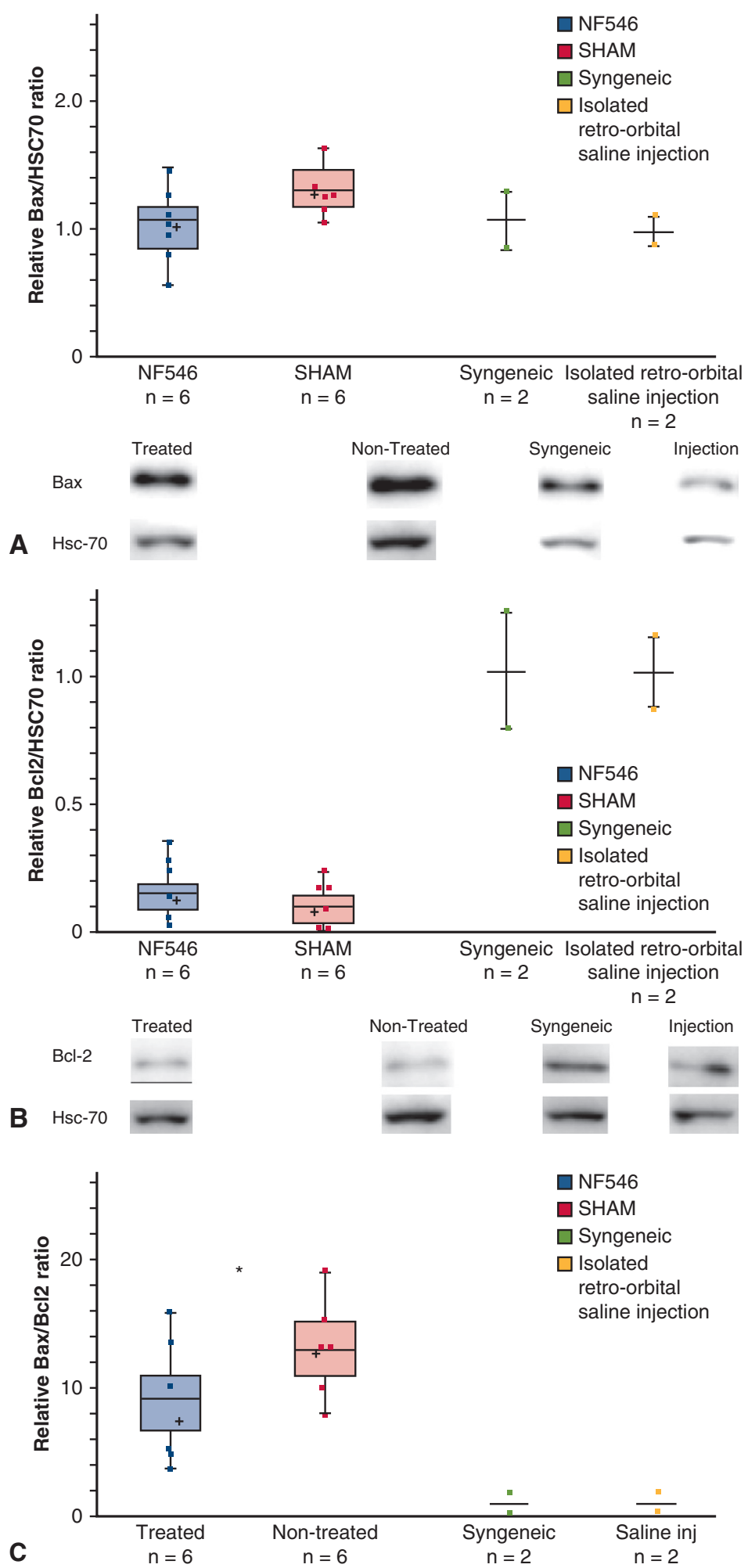

FIGURE 6. Quantification of apoptotic markers. Comparison of Bax/HSC70 (A), Bcl-2/HSC70 (B), and relative Bax/Bcl2 ratio (C) at day 5 in treated allografts (NF546; $n=6$ ), untreated allografts (SHAM; $n=6$ ), syngeneic grafts $(n=2)$, and mice undergoing isolated retro-orbital injection of vehicle $(\mathrm{n}=2)$. Data are presented as box-and-whiskers plots where the upper and lower borders of the box represent the upper and lower quartiles, the horizontal line inside the box represents the median, the upper and lower whiskers represent the maximum and minimum values of nonoutliers, and the + sign represents the mean. ${ }^{*} \mathrm{P} 2 \mathrm{Y} 11 \mathrm{R}$ agonist-treated allografts (blue) demonstrated a significantly lower Bax/Bcl-2 ratio compared with untreated allografts (red). $* P<.05$. 


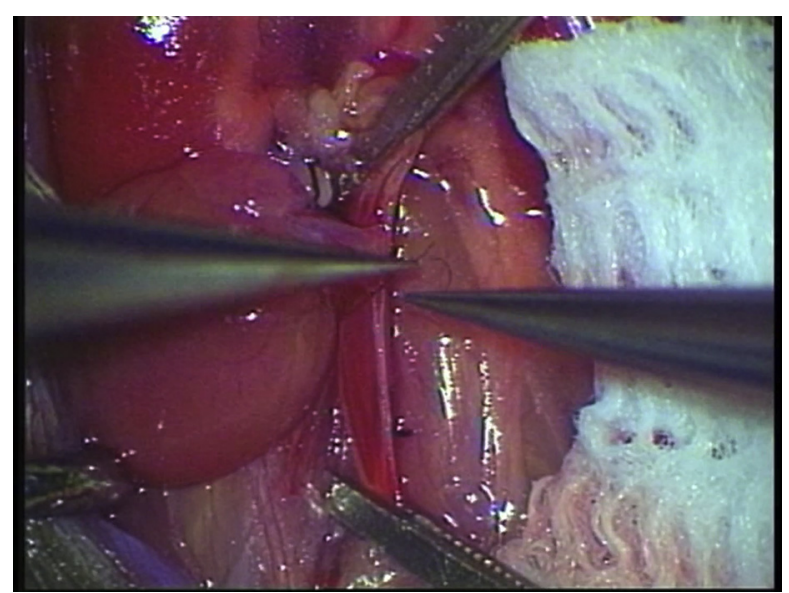

VIDEO 1. The main outcomes of the study and the technique of mouse heterotopic heart transplantation are described. Video available at: https://www.jtcvs.org/article/S0022-5223(18)33452-4/fulltext.

important regulator of immune system activation through P2Rs stimulation. ${ }^{3,24,25}$ It was shown that activating P2Rs by extracellular ATP upregulates maturation markers in dendritic cells and triggers a shift in cytokine production upon LPS or CD40L stimulation. ${ }^{31}$ Indeed, we and others previously reported that extracellular ATP modulated response of dendritic cells through a P2Y11R-dependent maturation toward a tolerogenic phenotype in decreasing proinflammatory cytokine release., ${ }^{9,10}$ Consistent with previous published data from our laboratory, we found that P2Y11R stimulation drove a decrease of IL-12 and IFN $\gamma$ production, while increasing the production of IL-10. ${ }^{10}$

In addition to the modulation of the inflammatory response, our data suggest a novel cardioprotective role of P2Y11R through a reduction of apoptosis. We showed that conditioning with P2Y11R specific agonist NF546 decreased the production of IL-33, whereas at day 5 posttransplant, caspase-3 activity and $\mathrm{Bax} / \mathrm{Bcl}-2$ ratio were attenuated compared with control. These data are consistent with several reports suggesting that multiple P2 receptors on macrophages may be involved in the modulation of apoptosis because dying cells are known to release nucleotides and other DAMPs. ${ }^{5,32-34}$ Elliott and colleagues ${ }^{32}$ reported that $\mathrm{P} 2 \mathrm{X} 7$ receptor stimulation resulted in "flipping" of membrane phosphatidylserine from the inner to the outer leaflet, so that it was exposed at the cell surface of lymphocytes. This modified lipid distribution altered the activity of at least 3 distinct membrane proteins and resulted in apoptosis downregulation. ${ }^{32}$ More recently, P2Y6 activation by uridine 5 '-diphosphate was implicated in phagocytosis of apoptotic bodies in microglial cells. ${ }^{33}$ Even if $\mathrm{P} 2 \mathrm{Y} 11 \mathrm{R}$ is expressed in macrophages, ${ }^{34}$ its precise function in modulating apoptosis remains to determine. Recent evidence suggests that extracellular nicotinic acid adenine dinucleotide phosphate signaling at the P2Y11-like receptor affords significant cardioprotection against I/R injury and induces prosurvival protein kinase activation. ${ }^{16}$

\section{Study Limitations}

A recent review ${ }^{20}$ provided criticism of publications focusing on P2Y11R expression and function. Indeed, the lack of a P2Y11 gene orthologue in the murine genome prevents the generation of knockout mice, which have been helpful for defining the roles of other P2Y receptors. According to recommended methods, ${ }^{20}$ we assessed P2Y11R expression by Western blot and function by using specific agonist and antagonist. We found in our in vitro work that the use of P2Y11R agonist NF546 provides H9c2 cell protection, similar to that observed in human cardiomyocytes, ${ }^{27}$ which was abolished by the specific antagonist NF340. These results, consistent with those found by others, ${ }^{16,21}$ support the existence of a P2Y11-like receptor in rodent.

\section{Future Directions}

Our results confirm the beneficial role of P2Y11-like receptor stimulation in cardiac allograft conditioning through 3 main mechanisms: protection against oxidative stress, modulation of the inflammatory response, and reduction of apoptosis (Central Figure). Because current immunosuppressive regimens are still associated with multiple complications, including cancer, opportunistic infections, diabetes mellitus, kidney failure, and hypertension, ${ }^{29}$ we think that short-term P2Y11R targeting at the time of transplantation may prove to be valuable in the clinical setting by promoting cardiac transplant tolerance and preventing rejection lesions. In addition, P2Y11R should be nonthrombogenic. It was reported that stimulation of human coronary artery endothelial cells by UTP/ATP increased the mRNA level of tissue factor. This tissue factor upregulation was inhibited by suramin or P2Y2 receptor silencing with small interfering RNA, but not by NF157, a P2Y11-selective antagonist. These data suggested a role of P2Y2 receptor, and not P2Y11R, in mediating tissue factor expression. ${ }^{35}$

\section{CONCLUSIONS}

Our results suggest a novel cardioprotective role of P2Y11R in the setting of cardiac transplantation. P2Y11like receptor stimulation by its specific agonist NF546 protects against $\mathrm{H} / \mathrm{R}$ injuries, improves allograft survival, and decreases rejection lesions. These data suggest that therapeutic interventions using pharmacologic conditioning with P2Y11R agonists could provide beneficial effects after cardiac transplantation in humans and improve patients' outcomes.

\section{Conflict of Interest Statement}

Authors have nothing to disclose with regard to commercial support. 


\section{References}

1. Reimer KA, Lowe JE, Rasmussen MM, Jennings RB. The wavefront phenomenon of ischemic cell death: myocardial infarct size vs duration of coronary occlusion in dogs. Circulation. 1977;56:786-94.

2. Yellon DM, Hausenloy DJ. Myocardial reperfusion injury. N Engl J Med. 2007; 357:1121-35.

3. Rubartelli A, Lotze MT. Inside, outside, upside down: damage-associated molecular- pattern molecules (DAMPs) and redox. Trends Immunol. 2007;28:429-36.

4. Burnstock G. Introduction to purinergic signalling in the brain. In: Advances in Experimental Medicine and Biology, 986. Springer Netherlands; 2013:1-12.

5. Erlinge D, Burnstock G. P2 receptors in cardiovascular regulation and disease. Purinergic Signal. 2008;4:1-20.

6. Alkayed F, Kashimata M, Koyama N, Hayashi T, Tamura Y, Azuma Y. P2Y11 purinoceptor mediates the ATP-enhanced chemotactic response of rat neutrophils. J Pharmacol Sci. 2012;120:288-95.

7. Parzych K, Zetterqvist AV, Wright WR, Kirkby NS, Mitchell JA, Paul-Clark MJ Differential role of pannexin-1/ATP/P2X7 axis in IL-1 $\beta$ release by human monocytes. FASEB J. 2017:31:2439-45.

8. Karmakar M, Katsnelson MA, Dubyak GR, Pearlman E. Neutrophil P2X7 receptors mediate NLRP3 inflammasome-dependent IL-1 $\beta$ secretion in response to ATP. Nat Commun. 2016;7:10555.

9. Wilkin F, Duhant X, Bruyns C, Suarez-Huerta N, Boeynaems JM, Robaye B. The P2Y11 receptor mediates the ATP-induced maturation of human monocytederived dendritic cells. J Immunol. 2001;166:7172-7.

10. Chadet S, Ivanes F, Benoist L, Salmon-Gandonnière C, Guibon R, VelgeRoussel F, et al. Hypoxia/reoxygenation inhibits P2Y11 receptor expression and its immunosuppressive activity in human dendritic cells. J Immunol. 2015; 195:651-60.

11. Yusuf S, Zhao F, Mehta SR, Chrolavicius S, Tognoni G, Fox KK. Effects of clopidogrel in addition to aspirin in patients with acute coronary syndromes without ST-segment elevation. $N$ Engl J Med. 2001;345:494-502.

12. Communi D, Govaerts C, Parmentier M, Boeynaems JM. Cloning of a human purinergic $\mathrm{P} 2 \mathrm{Y}$ receptor coupled to phospholipase $\mathrm{C}$ and adenylyl cyclase. J Biol Chem. 1997;272:31969-73.

13. Communi D, Robaye B, Boeynaems JM. Pharmacological characterization of the human P2Y11 receptor. Br J Pharmacol. 1999;128:1199-206.

14. Forrester T, Williams CA. Release of adenosine triphosphate from isolated adult heart cells in response to hypoxia. J Physiol. 1977;268:371-90.

15. Williams CA, Forrester T. Possible source of adenosine triphosphate released from rat myocytes in response to hypoxia and acidosis. Cardiovasc Res. 1983; 17:301-12.

16. Djerada Z, Millart HH. Intracellular NAADP increase induced by extracellular NAADP via the P2Y11-like receptor. Biochem Biophys Res Commun. 2013; 436:199-203.

17. Niimi M. The technique of heterotopic cardiac transplantation in mice: experience of 3000 operations by one surgeon. J Heart Lung Transplant. 2001;20: 1123-8.

18. Berry GJ, Burke MM, Andersen C, Bruneval P, Fedrigo M, Fishbein MC, et al. The 2013 ISHLT working formulation for the standardization of nomenclature in the pathologic diagnosis of antibody-mediated rejection in heart transplantation. J Heart Lung Transplant. 2013;32:1147-62.

19. Schindelin J, Arganda-Carreras I, Frise E, Kaynig V, Longair M, Pietzsch T, et al. Fiji: an open-source platform for biological-image analysis. Nat Methods. 2012; 9:676-82.

20. Dreisig K, Kornum BR. A critical look at the function of the P2Y11 receptor. Purinergic Signal. 2016;12:427-37.
21. Balogh J, Wihlborg A-KK, Isackson H, Joshi BV, Jacobson KA, Amer A, et al. Phospholipase C and cAMP-dependent positive inotropic effects of ATP in mouse cardiomyocytes via P2Y11-like receptors. J Mol Cell Cardiol. 2005;39: 223-30.

22. Amisten S, Melander O, Wihlborg AK, Berglund G, Erlinge D. Increased risk of acute myocardial infarction and elevated levels of $\mathrm{C}$-reactive protein in carriers of the Thr-87 variant of the ATP receptor P2Y11. Eur Heart J. 2007;28:13-8.

23. Bodin P, Burnstock G. Synergistic effect of acute hypoxia on flow-induced release of ATP from cultured endothelial cells. Experientia. 1995;51:256-9.

24. Riteau N, Gasse P, Fauconnier L, Gombault A, Couegnat M, Fick L, et al. Extracellular ATP is a danger signal activating P2X7 receptor in lung inflammation and fibrosis. Am J Respir Crit Care Med. 2010;182:774-83.

25. Iyer SS, Pulskens WP, Sadler JJ, Butter LM, Teske GJ, Ulland TK, et al. Necrotic cells trigger a sterile inflammatory response through the Nlrp3 inflammasome. Proc Natl Acad Sci U S A. 2009;106:20388-93.

26. Meis S, Hamacher A, Hongwiset D. 1-phenylene-carbonylimino-3, 1-(4-methylphenylene)-carbonylimino))-bis (1,3-xylene- $\alpha, \alpha^{\prime}$-diphosphonic acid) tetrasodium salt] is a non-nucleotide P2Y11 agonist. J Pharmacol Exp Ther. 2010; $332: 238-47$.

27. Lefort C, Benoist L, Chadet S, Piollet M, Heraud A, Babuty D, et al. Stimulation of P2Y11 receptor modulates cardiac fibroblasts secretome toward immunomodulatory and protective roles after Hypoxia/reperfusion injury. J Mol Cell Cardiol. 2018;121:212-21

28. Baines CP, Song CX, Zheng YT, Wang GW, Zhang J, Wang OL, et al. Protein kinase Cepsilon interacts with and inhibits the permeability transition pore in cardiac mitochondria. Circ Res. 2003;92:873-80.

29. Lund LH, Khush KK, Cherikh WS, Goldfarb S, Kucheryavaya AY, Levvey BJ, et al; for the International Society for Heart and Lung Transplantation. Registry of the International Society for Heart and Lung Transplantation: thirty-fourth adult heart transplantation report-2017; Focus theme: allograft ischemic time. J Heart Lung Transplant. 2017;36:1037-47.

30. Erickson RP. Mouse models of human genetic disease: which mouse is more like a man? Bioassays. 1996;18:993-8.

31. La Sala A, Ferrari D, Corinti S, Cavani A, Di Virgilio F, Girolomoni G. Extracellular ATP induces a distorted maturation of dendritic cells and inhibits their capacity to initiate Th1 responses. J Immunol. 2001;166:1611-7.

32. Elliott JI, Surprenant A, Marelli-Berg FM, Cooper JC, Cassady-Cain RL, Wooding $\mathrm{C}$, et al. Membrane phosphatidylserine distribution as a nonapoptotic signalling mechanism in lymphocytes. Nat Cell Biol. 2005;7: 808-16.

33. Koizumi S, Shigemoto-Mogami Y, Nasu-Tada K, Shinozaki Y, Ohsawa K, Tsuda M, et al. UDP acting at P2Y6 receptors is a mediator of microglial phagocytosis. Nature. 2007;446:1091-5.

34. Sakaki H, Tsukimoto M, Harada H, Moriyama Y, Kojima S. Autocrine regulation of macrophage activation via exocytosis of ATP and activation of P2Y11 receptor. PLoS One. 2013;8:e59778.

35. Ding L, Ma W, Littmann T, Camp R, Shen J. The P2Y2 nucleotide receptor mediates tissue factor expression in human coronary artery endothelial cells. J Biol Chem. 2011;286:27027-38.

Key Words: allograft, animal model, echocardiography, experimental surgery, heart transplantation, inflammatory mediators, immunochemistry, immunology, postconditioning, rejection 
TABLE E1. Inflammation decrease in P2Y11R agonist-treated allografts

\begin{tabular}{lrcc}
\hline $\begin{array}{c}\text { Concentration } \\
(\mathbf{p g} / \mathbf{m L}) \text { mean } \pm \text { SEM }\end{array}$ & $\begin{array}{c}\text { NF546 } \\
(\mathbf{n}=\mathbf{6})\end{array}$ & $\begin{array}{c}\text { SHAM } \\
(\mathbf{n}=\mathbf{6})\end{array}$ & $\boldsymbol{P}$ value \\
\hline IFN $\gamma$ & $98.6 \pm 6.4$ & $984.2 \pm 43.4$ & $<.001$ \\
IL4 & $0.9 \pm 0.1$ & $8.1 \pm 2.4$ & NS \\
\hline IL5 & $6.1 \pm 1.9$ & $1.8 \pm 1.1$ & NS \\
IL6 & $185.2 \pm 9.4$ & $221.7 \pm 8.9$ & NS \\
\hline IL10 & $17.3 \pm 3.1$ & $9.3 \pm 2.4$ & $<.05$ \\
\hline IL12 (p70) & $1.9 \pm 0.4$ & $8.4 \pm 1.8$ & $<.05$ \\
\hline IL13 & $13.1 \pm 2.7$ & $16.5 \pm 3.1$ & NS \\
IL17A & $2.2 \pm 0.3$ & $2.5 \pm 0.6$ & NS \\
\hline IL17F & $1.1 \pm 0.4$ & $2.2 \pm 0.6$ & NS \\
IL22 & $5.0 \pm 1.1$ & $4.6 \pm 0.6$ & NS \\
\hline IL33 & $11.2 \pm 2.3$ & 13.1 & NS \\
TNF- $\alpha$ & $16.1 \pm 3.1$ & $12.9 \pm 2.7$ & NS \\
\hline
\end{tabular}

Serum levels (pg/mL) of IFN $\gamma$, IL4, IL5, IL6, IL10, IL12 (p70), IL13, IL17A, IL17F,

IL22, IL33, and TNF $\alpha$ measured at day 5 after transplantation. Data correspond to mean \pm SEM of $n=12$ mice, treated with P2Y11R agonist (NF546; $n=6)$ or vehicle (SHAM; $\mathrm{n}=6$ ). At day 5, P2Y11R agonist-treated mice showed significantly lower serum levels of proinflammatory cytokines IFN $\gamma$ and IL-12, and higher level of antiinflammatory cytokine IL-10 compared with vehicle. SEM, Standard error of the mean; $I F N$, interferon; $N S$, not significant; $I L$, interleukin; $T N F$, tumor necrosis factor. 